\title{
SINGULAR SETS AND REMAINDERS
}

BY

\author{
GEORGE L. CAIN, RICHARD E. CHANDLER
}

AND GARY D. FAULKNER

\begin{abstract}
This paper characterizes the singular sets of several traditional classes of continuous mappings associated with compactifications. By relating remainders of compactifications to singular sets of mappings with compact range, new results are obtained about each.
\end{abstract}

All spaces to be considered here are locally compact and Hausdorff and all functions are continuous. The compact subsets of a space $Z$ will be denoted by $\mathcal{K}_{Z}$. For $p \in Z, \mathscr{K}(p)\left(\mathcal{T}_{\kappa}(p)\right)$ denotes the family of open neighborhoods (open neighborhoods with compact closure) of $p$. For a mapping $f: X \rightarrow Y$, Cain [2] defined the singular set of $f$ to be

$$
\mathcal{S}(f)=\left\{p \in Y \mid \forall U \in \mathcal{K}(p), \exists F \in \mathcal{K}_{Y}, p \in F \subseteq U \text { and } f^{-1}(F) \notin \mathcal{K}_{X}\right\} .
$$

In a later paper [3], he further explored the nature of $\delta(f)$. Whyburn [14] gave an alternate definition.

Various authors have seen methods of constructing a compactification of $X$ given a map $f: X \rightarrow Y$ where $Y$ is compact. Perhaps the earliest (in the context of this paper) was Loeb [9]. Others who should be mentioned are Steiner and Steiner [11], [12], Magill [10], Blakley, Gerlits and Magill [1], and Choo [7]. Chandler and Tzung [6] defined the remainder induced by $f$ to be

$$
\mathcal{L}(f)=\cap\left\{\operatorname{cl}_{Y} f(X \backslash F) \mid F \in \mathscr{K}_{X}\right\}
$$

and proved that (whenever $Y$ is compact) there is a compactification $\alpha X$ with $\alpha X \backslash X$ homeomorphic to $\mathscr{L}(f)$.

We show here that $\mathcal{S}(f)$ and $\mathcal{L}(f)$ are the same set. We can then use what was previously known about singular sets to obtain results about remainders and vice versa. We will also explore this set for three types of mappings commonly encountered in studying compactifications: (i) the composition of a mapping of $X$ into $Y$ and the inclusion of $Y$ into a compactification of $Y$; (ii) the composition of a mapping of $X$ into $Y$ and a quotient mapping of $Y$; and (iii) the evaluation mapping into a product generated by the inclusion of $X$ into a compactification and by a mapping of $X$ into a compact space $K$.

Received by the editors April 9, 1980 and, in revised form, October 1, 1980.

AMS (MOS) subject classifications (1970). Primary 54D35, 54D40, 54C10.

Key words and phrases. Compactification, remainder, singular set.

(C) 1981 American Mathematical Society 0002-9947/81/0000-0508/\$03.75 
1. The basic result. We first define the set $\mathcal{S}^{\prime}(f)$ to be

$$
\left\{p \in Y \mid \forall U \in \mathscr{K}_{\kappa}(p), f^{-1}(\bar{U}) \notin \mathscr{K}_{X}\right\},
$$

and the set $\bar{\delta}(f)$ to be

$$
\left\{p \in Y \mid \forall U \in \mathcal{N}(p), \overline{f^{-1}(U)} \notin \mathcal{K}_{X}\right\} .
$$

An obviously equivalent definition of $\varrho(f)$ is the set

$$
\left\{p \in Y \mid \forall U \in \Re(p), \forall F \in \mathcal{K}_{X}, U \cap f(X \backslash F) \neq \varnothing\right\} .
$$

THEOREM 1.1. $\delta(f)=\mathcal{L}(f)=\delta^{\prime}(f)=\bar{\delta}(f)$.

Proof. If $p \notin \mathcal{L}(f)$ there is a $U \in \mathcal{R}(p)$ and an $F \in \mathcal{K}_{X}$ for which $U \cap$ $f(X \backslash F)=\varnothing$. Then $f^{-1}(U) \subseteq F$. For any $K \in \mathcal{K}_{Y}$ with $p \in K \subseteq U$ we have $f^{-1}(K)$ is a closed subset of $F$ and is therefore compact. It follows that $p \notin \delta(f)$. Thus $\mathcal{S}(f) \subseteq \mathcal{L}(f)$.

Next, suppose $p \notin \delta^{\prime}(f)$. Then there exists $U \in \mathscr{T}_{\kappa}(p)$ for which $f^{-1}(\bar{U})$ is compact. $f\left(X \backslash f^{-1}(\bar{U})\right)=f(X) \cap(Y \backslash \bar{U})$. Now $p \in U$ and $U \cap[f(X) \cap$ $(Y \backslash \bar{U})]=\varnothing$ so that $p \notin \mathcal{L}(f)$. We conclude that $\mathcal{L}(f) \subseteq \mathcal{S}^{\prime}(f)$.

If $p \in \mathcal{S}^{\prime}(f)$ and $U \in \mathcal{X}(p)$ choose $V \in \Re_{\kappa}(p)$ with $\bar{V} \subseteq U$. Then $\overline{f^{-1}(U)} \supseteq$ $f^{-1}(\bar{V})$ so that if $\overline{f^{-1}(U)}$ were compact then $f^{-1}(\bar{V})$ would be also. It follows that $p \in \bar{\delta}(f)$.

Finally, suppose $p \in \overline{\mathcal{S}}(f)$ and $U \in \mathcal{R}(p)$. Choose $V \in \Re_{\kappa}(p)$ with $\bar{V} \subseteq U$. Then $\overline{f^{-1}(V) \subseteq f^{-1}(\bar{V}) \text { and } f^{-1}(V)}$ is not compact. Thus $f^{-1}(\bar{V})$ is not compact. We have determined an element of $\mathscr{K}_{Y}$ contained in $U$ whose inverse image under $f$ is not compact. Thus, $p \in \delta(f)$.

Lemma 1.2. If $f(X)$ is dense in $Y$, then $Y \backslash f(X) \subseteq \delta(f)$.

Proof. For $p \in Y \backslash f(X)$ there is a net $\left\{x_{\lambda}\right\}$ in $X$ for which $\left\{f\left(x_{\lambda}\right)\right\} \rightarrow p$. Let $U \in \Re_{\kappa}(p) .\left\{f\left(x_{\lambda}\right)\right\}$ is eventually in $U$. If $f^{-1}(\bar{U})$ were compact, $\left\{x_{\lambda}\right\}$ would have a cluster point $x$ in $f^{-1}(\bar{U})$. But then $f(x)$ would be a cluster point of $\left\{f\left(x_{\lambda}\right)\right\}$, contradicting the fact that $\left\{f\left(x_{\lambda}\right)\right\} \rightarrow p \notin f(X)$. Thus, for an arbitrary $U \in \mathscr{T}_{\kappa}(p)$, $f^{-1}(\bar{U}) \notin \mathcal{K}_{X}$. We conclude that $Y \backslash f(X) \subseteq \mathcal{S}(f)$.

2. Composition with inclusion maps. We next consider the singular set of the composition of two mappings when the second map is the inclusion of a space in one of its compactifications. We use the following notation: If $Y$ is a space and $\alpha Y$ is one of its compactifications, then $\alpha: Y \rightarrow \alpha(Y) \subseteq \alpha Y$ is the inclusion. However, we follow the standard abuse of this convention by using $\alpha Y \backslash Y$ to denote $\alpha Y \backslash \alpha(Y)$.

THEOREM 2.1. Suppose $f: X \rightarrow Y$ is a mapping with $f(X)$ dense in $Y$. Then

$$
\delta(\alpha \circ f)=\delta(\alpha) \cup \alpha(\delta(f))=(\alpha Y \backslash Y) \cup \alpha(\delta(f)) .
$$

Proof. That $\delta(\alpha)=\alpha Y \backslash Y$ follows immediately from 1.2 and the fact that for each point $y \in Y=\alpha(Y)$ there is $U \in \Re_{\kappa}(y)$ such that $\bar{U} \cap(\alpha Y \backslash Y)=\varnothing$. $\alpha^{-1}(\bar{U})=\bar{U}$ is compact. 
Now, suppose $p \in \mathcal{S}(\alpha)=\alpha Y \backslash Y=\mathcal{S}^{\prime}(\alpha)$. Then for each $U \in \mathcal{R}_{\kappa}(p)$ we have $\alpha^{-1}(\bar{U}) \notin \mathscr{K}_{Y}$. It follows that $(\alpha \circ f)^{-1}(\bar{U}) \notin \mathscr{K}_{X}$ since otherwise $f\left((\alpha \circ f)^{-1}(\bar{U})\right)=$ $\alpha^{-1}(\bar{U}) \cap f(X)$ would be compact. Since $\overline{\alpha^{-1}(U)} \cap f(X)$ is a closed subset of $\alpha^{-1}(\bar{U}) \cap f(X)$, we would then have that $\overline{\alpha^{-1}(U)} \cap f(X)$ is compact. Now $\alpha^{-1}(U)$ $\cap f(X) \subseteq \overline{\alpha^{-1}(U)} \cap f(X)$ so that $\overline{\alpha^{-1}(U) \cap f(X)}=\overline{\alpha^{-1}(U)}$ would be compact, contradicting the fact that $p \in \mathcal{S}(\alpha)=\bar{\delta}(\alpha)$.

Next, suppose that $p=\alpha(q)$ with $q \in \mathcal{S}(f)$. For any $U \in \mathscr{N}(p)$ we have $\alpha^{-1}(U) \in \mathscr{X}(q)$ so there exists $F \in \mathcal{K}_{Y}$ with $f^{-1}(F) \notin \mathcal{K}_{X}$ and $q \in F \subseteq \alpha^{-1}(U)$. Now $p \in \alpha(F) \subseteq U$ and $\alpha(F) \in \mathscr{K}_{\alpha Y}$. If $(\alpha \circ f)^{-1}(\alpha(F))$ were compact then $f^{-1}(F)$ would also since it is a closed subset. Thus, $p \in \mathcal{S}(\alpha \circ f)$ and so $\alpha(\mathcal{S}(f)) \subseteq$ $\delta(\alpha \circ f)$.

Finally, suppose $p \in \mathcal{S}(\alpha \circ f)$. We can assume $p \in \alpha(Y)$ for otherwise we are through. Let $p=\alpha(q)$ and suppose $U \in \Re_{\kappa}(q)$. Then $\alpha(U) \in \Re_{\kappa}(p)$ and so $(\alpha \circ f)^{-1}(\overline{\alpha(U)})=f^{-1}(\bar{U})$ is not compact. But this says that $q \in \mathcal{S}(f)$. Thus we have shown that $\delta(\alpha \circ f) \subseteq \delta(\alpha) \cup \alpha(\delta(f))$.

We will use the notation $f: X \Rightarrow Y$ to denote a continuous map from $X$ onto $Y$. If $\delta(f) \neq \varnothing$ for such a map, we say that $f$ is singular. Otherwise, $f$ is nonsingular (= compact). We are now in a position to use the preceding theorem to obtain an important corollary which first appeared in [1]. Then we use the corollary to obtain several results concerning the existence or nonexistence of singular and nonsingular maps between specific spaces. $R(X)$ will denote the set of remainders of $X$, i.e. the set of all $\alpha X \backslash X$ where $\alpha X$ is a compactification of $X$.

COROLlaRY 2.2. If there exists a nonsingular map $f: X \Rightarrow Y$ then $\mathscr{R}(Y) \subseteq \Re(X)$.

Proof. For any compactification $\alpha Y$ of $Y$ we have $\mathcal{L}(\alpha \circ f)=\delta(\alpha \circ f)=$ $\alpha Y \backslash Y \cup \alpha(\mathcal{S}(f))=\alpha Y \backslash Y$. But $\mathcal{L}(\alpha \circ f)$ is a remainder of $X$.

Corollary 2.3. Every map $f: \mathbf{R}^{n} \Rightarrow \mathbf{R}^{1}$ is singular if $n>1$.

Proof. Since $\mathbf{R}^{1}$ has a 2-point compactification and $\mathbf{R}^{n}$ does not if $n>1$, $\Re\left(\mathbf{R}^{1}\right) \not \subset \Re\left(\mathbf{R}^{n}\right)$.

Corollary 2.4. For $n>1$, no map $f: \mathbf{R}^{n} \Rightarrow \mathbf{R}^{1}$ is closed.

Proof. If such a map existed, $\delta(f)$ would be scattered $[2,3.7]$ or empty. The second case is not possible by the preceding corollary. In the first case, $\delta(\omega \circ f)$ would be disconnected, giving $\mathbf{R}^{n}$ a disconnected remainder. ( $\omega \mathbf{R}^{1}$ denotes the 1-point compactification of $\mathbf{R}^{\mathbf{1}}$.)

COROLlaRy 2.5. Let $f: \mathbf{R}^{m} \Rightarrow \mathbf{R}^{n}$. The only possible cardinalities for $\delta(f)$ are 0 and $c$ if $m>1$. If $m=1$ the only possibilities are 0,1 , or $c$.

Proof. There are no remainders of $\mathbf{R}^{m}$ with cardinalities greater than one and less than $c$ if $m>1$. By composing $f$ with the inclusion of $\mathbf{R}^{n}$ into its 1-point compactification we obtain a map of $\mathbf{R}^{m}$ into a compact space $\omega \mathbf{R}^{n}$ whose singular set has one more element in it than $\mathcal{S}(f)$ had. The first assertion follows easily. If 
$m=1$, the remainders of $\mathbf{R}^{m}$ do not have cardinalities greater than two and less than $c$. The second assertion now follows from the same type of reasoning as above.

It is clear that the existence of a continuous map $f: X \rightarrow Y=\overline{f(X)}$ is not sufficient to obtain $\Re(Y) \subseteq \Re(X)$. (Consider the projection of $\mathbf{R}^{2}$ onto $\mathbf{R}^{1}$.) There is, however, a class of remainders which is preserved, in this sense, under continuous maps. We will say that $K \in \mathcal{R}(X)$ is a singular remainder if there is a map $f$ : $X \rightarrow K$ so that $\delta(f)=K$. We will denote the collection of singular remainders of $X$ by $\mathcal{R}(X)$. For example, $\delta \mathscr{R}(\mathbf{N})=\{K \mid K$ is compact and separable $\}, \mathcal{R}(\mathbf{R})$ $=\{K \mid K$ is a weak Peano space $\}$ (a weak Peano space is a compact space which contains a dense continuous image of $\mathbf{R})$. In view of the following theorem, it seems that it would be desirable to characterize the singular remainders of spaces of interest.

THEOREM 2.6. If $f: X \rightarrow Y=\overline{f(X)}$ and $g: Y \rightarrow K$ with $\delta(g)=K$, then $\delta(g \circ f)$ $=K$.

Proof. Suppose $(g \circ f)^{-1}(\bar{U})$ were compact for some nonempty open set $U \subseteq K$. Then it follows that each of the following sets would be a compact subset of $Y$ :

(i) $f\left((g \circ f)^{-1}(\bar{U})\right)=g^{-1}(\bar{U}) \cap f(X)$,

(ii) $\overline{g^{-1}(U)} \cap f(X)$,

(iii) $\overline{g^{-1}(U) \cap f(X)}$,

(iv) $\overline{g^{-1}(U)}$.

However, since $\bar{\delta}(g)=\delta(g)=K$, this last set cannot be compact. Thus, $(g \circ f)^{-1}(\bar{U})$ is not compact so that $\delta(g \circ f)=K$.

Corollary 2.7. If $f: X \rightarrow Y=\overline{f(X)}$, then $\delta \Re(Y) \subseteq \delta \Re(X)$.

3. Composition with quotient maps. We next consider the singular set of the composition of two maps when the second is a quotient mapping.

Lemma 3.1. Suppose $f: X \rightarrow Y$ and $\phi: Y \rightarrow Y / R$ where $R$ is an equivalence relation on $Y$ for which $Y / R$ is locally compact, Hausdorff and has the property that for $p \notin \mathcal{S}(f)$ we have $(p, q) \in R$ if and only if $p=q$. Then $\delta(\phi \circ f)=\phi(\mathcal{S}(f))$.

Proof. The proof that $\phi(\delta(f)) \subseteq \delta(\phi \circ f)$ is the same as the proof showing that $\alpha(\delta(f)) \subseteq \delta(\alpha \circ f)$ in 2.1. Suppose $p \in \mathcal{S}(\phi \circ f)$ and that $p \notin \phi(\delta(f))$. Then $p=\phi(q)$ and $q \notin \delta(f)$. There exists $V \in \mathcal{T}_{\kappa}(q)$ such that $f^{-1}(\bar{V}) \in \mathcal{K}_{X}$. Also, there exists $W \in \mathcal{T}_{\kappa}(q)$ with $W \cap \delta(f)=\varnothing$. Let $U=V \cap W$. Then $f^{-1}(\bar{U}) \in$ $\mathcal{K}_{X}$ and $\phi^{-1}(\phi(\bar{U}))=\bar{U}$. On the one hand we have $(\phi \circ f)^{-1}(\phi(\bar{U}))=f^{-1}(\bar{U}) \in \mathcal{K}_{X}$. However, $\phi(U)$ is a member of $\mathcal{T}_{\kappa}(p)$ and $\overline{\phi(U)}=\phi(\bar{U})$; consequently, $(\phi \circ f)^{-1}(\overline{\phi(U)}) \notin \mathcal{K}_{X}$. This contradiction insures that $p \in \mathcal{S}(\phi \circ f)$ implies that $p \in \phi(\mathcal{S}(f))$.

We use this lemma to strengthen a result of Cain [5].

THEOREM 3.2. Suppose $f: X \rightarrow f(X)=Y$ and $\delta(f)$ has $k$ components, each compact. If $Y$ has an $n$-point compactification $\alpha Y$ then $X$ has an $(n+k)$-point compactification. 
Proof. Suppose $\delta(f)=K_{1} \cup \cdots \cup K_{k}$, each compact. Define an equivalence relation $R$ on $\alpha Y:(p, q) \in R$ if and only if $p=q$ or $p, q \in K_{i}$ for some $i, 1 \leqslant i<$ $k$.

$\alpha Y / Y$ is compact Hausdorff and we have $f: X \rightarrow Y, \alpha: Y \rightarrow \alpha Y, \phi: \alpha Y \rightarrow$ $\alpha Y / R$. By 3.1

$$
\begin{aligned}
\delta(\phi \circ \alpha \circ f) & =\phi(\delta(\alpha \circ f))=\phi(\delta(\alpha) \cup \alpha(\delta(f))) \\
& =\phi(\alpha Y \backslash Y) \cup\left\{p_{1}, \ldots, p_{k}\right\}
\end{aligned}
$$

which has $n+k$ points.

4. Evaluation maps. If $\alpha X$ is a compactification of $X$ and $f: X \rightarrow K$, where $K$ is compact, then the map $e: X \rightarrow \alpha X \times K$ defined by $e(x)=(x, f(x))$ is an embedding and $e X=\operatorname{cl}(e(X))$ is a compactification of $X$. $\delta(e)$ will be $e X \backslash e(X)$, the remainder of $X$ in $e X$. Our immediate goal is to characterize $\delta(e)$. To do this, define

$$
E=\left\{p \in \alpha X \backslash X \mid \bar{f}\left(\pi^{-1}(p)\right) \text { is a singleton }\right\},
$$

where $\bar{f}: \beta X \rightarrow K$ is the standard extension of $f$ to $\beta X$ and $\pi: \beta X \rightarrow \alpha X$ is the usual quotient map. Let

$$
\begin{gathered}
\Gamma_{f}=\left\{(p, q) \in \alpha X \times K \mid p \in E \text { and }\{q\}=\bar{f}\left(\pi^{-1}(p)\right)\right\}, \\
\Delta_{f}=\left\{(p, q) \in \alpha X \times K \mid p \in(\alpha X \backslash X) \backslash E \text { and } q \in \bar{f}\left(\pi^{-1}(p)\right)\right\} .
\end{gathered}
$$

THEOREM 4.1. $\delta(e)=\Gamma_{f} \cup \Delta_{f}$.

Proof. We show this by proving that $e X \backslash e(X)=\Gamma_{f} \cup \Delta_{f}$. This we do by showing that $e(X) \cup \Gamma_{f} \cup \Delta_{f}$ is closed in $\alpha X \times K$ and that every point of $\Gamma_{f} \cup \Delta_{f}$ is a limit point of $e(X)$.

Let $\left\{\left(p_{\lambda}, q_{\lambda}\right)\right\}$ be an ultra net in $e(X) \cup \Gamma_{f} \cup \Delta_{f}$. Then, regardless of which of the sets $\left(p_{\lambda}, q_{\lambda}\right)$ is in, we have $q_{\lambda} \in \bar{f}\left(\pi^{-1}\left(p_{\lambda}\right)\right)$. Choose $z_{\lambda} \in \pi^{-1}\left(p_{\lambda}\right) .\left\{z_{\lambda}\right\}$ has a cluster point $z$ in $\beta X$ so that $\left\{p_{\lambda}\right\} \rightarrow p=\pi(z)$ and $\left\{q_{\lambda}\right\} \rightarrow q=\bar{f}(z)$. It follows that $q \in \bar{f}\left(\pi^{-1}(p)\right)$ so that $(p, q) \in e(X) \cup \Gamma_{f} \cup \Delta_{f}$.

Next, suppose $(p, q) \in \Gamma_{f} \cup \Delta_{f}$ and let $U \times V$ be a basic neighborhood of $(p, q)$ in $\alpha X \times K$. Let $O=\pi^{-1}(U) \cap \bar{f}^{-1}(V)$. Then $O$ is a nonempty subset of $\beta X$, and if we choose $x \in O$ then we have $e(x)=(x, f(x))=(x, \bar{f}(x)) \in \pi(O) \times \bar{f}(O) \subseteq U$ $\times V$. Thus each point of $\Gamma_{f} \cup \Delta_{f}$ is a limit point of $e(X)$.

We next give an example which was particularly revealing when we were developing Theorem 4.1. We let $X=\mathbf{R}^{1}, K=[-1,1]$ and $f: \mathbf{R}^{1} \rightarrow[-1,1]$ is given by $f(x)=\sin x . \quad \alpha \mathbf{R}^{1}$ is the compactification having $[-1,1]$ as a remainder obtained by embedding $\mathbf{R}^{1}$ in $\omega \mathbf{R}^{1} \times[-1,1]$ using the map $g: \mathbf{R}^{1} \rightarrow[-1,1]$ to give the second coordinate (the inclusion of $\mathbf{R}^{1}$ into $\omega \mathbf{R}^{1}$, the 1-point compactification, gives the first coordinate) where

$$
g(x)= \begin{cases}\sin x, & x>0 \\ \inf \{0, \sin x\}, & x<0\end{cases}
$$


We may indicate the relevant ideas in the following sketch of $\alpha \mathbf{R}^{1} \times[-1,1]$ :

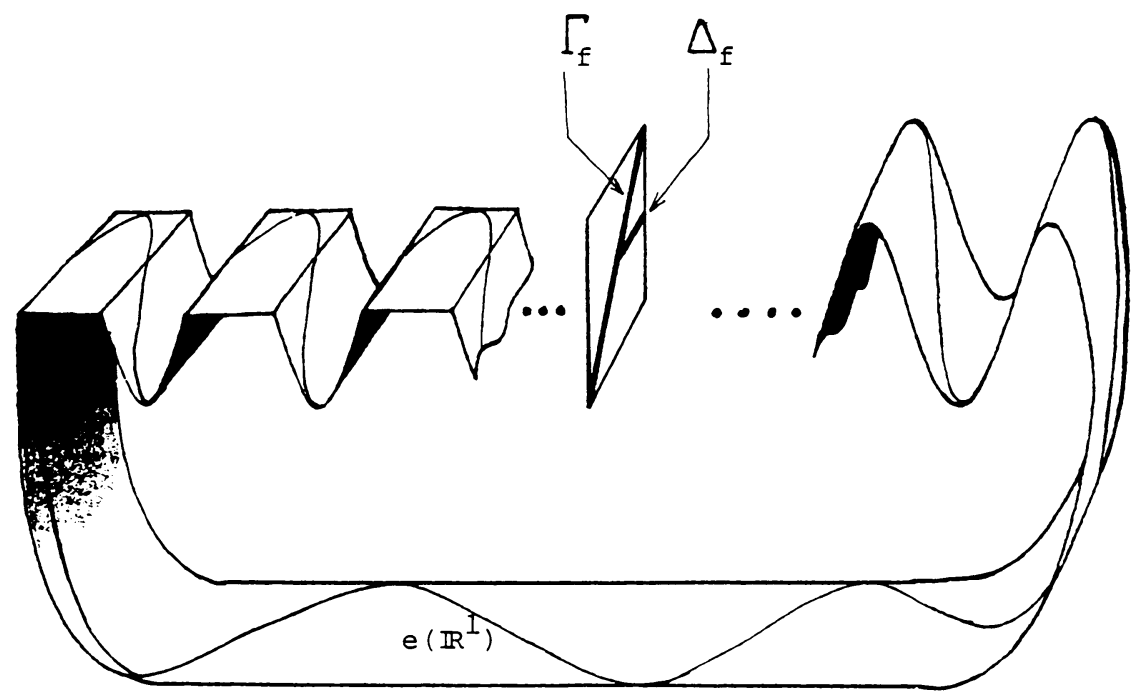

Notice that in this case, $\alpha \mathbf{R}^{1} \backslash \mathbf{R}^{1}=[-1,1]$ and that $E=[-1,0) \cup(0,1]$. $\bar{f}\left(\pi^{-1}(p)\right)$ is equal to $\{p\}$ and so $\Gamma_{f}=\{(p, p) \mid p \in E\}$. For $p=0 \in\left(\alpha \mathbf{R}^{1} \backslash \mathbf{R}^{1}\right) \backslash E$ we have $\bar{f}\left(\pi^{-1}(p)\right)=[0,1]$ and so $\Delta_{f}=\{(0, q) \mid q \in[0,1]\}=\{0\} \times[0,1]$.

Using 4.1 we generalize a result of Steiner and Steiner [11] which gives a sufficient condition for a compact space $K$ to be a remainder of $X$. We will call a compact space $K$ totally singular with respect to a compactification $\alpha X$ if there is a map $f: X \rightarrow K$ so that $f(U \cap X)$ is dense in $K$ for all $U$ which are neighborhoods of points in $\alpha X \backslash X$. For example, $[-1,1]$ is totally singular with respect to $\tau \mathbf{R}^{1}$, the 2-point compactification of $\mathbf{R}^{1}$, using $f(x)=\sin x$. Also, if $f: X \rightarrow K$ and $\delta(f)=$ $K$, then $K$ is totally singular with respect to $\omega X$, the 1-point compactification of $X$.

THEOREM 4.2. If $K$ is totally singular with respect to $\alpha X$ then $(\alpha X \backslash X) \times K$ is a remainder of $X$.

Proof. We will show that the hypothesis implies that $\Gamma_{f}=\varnothing$ by showing the set $E$ is empty. To do this we prove that $\bar{f}\left(\pi^{-1}(p)\right)$ is dense in $K$ for each $p \in \alpha X \backslash X$. It will follow that $\bar{f}\left(\pi^{-1}(p)\right)=K$ since it is compact. Let $O$ be any compact neighborhood in $K$. For each $U \in \mathcal{T}(p)$ select $x_{U} \in U$ for which $f\left(x_{U}\right) \in O$. Considered as a net in $\alpha X,\left\{x_{U}\right\} \rightarrow p$. Considered as a net in $\beta X,\left\{x_{U}\right\}$ has a cluster point $z$. Clearly, $\pi(z)=p$ and since $O$ is compact, $\bar{f}(z) \in O$. We have shown that $\bar{f}\left(\pi^{-1}(p)\right) \cap O \neq \varnothing$ whenever $O$ is a compact neighborhood in $K$. We conclude that $\bar{f}\left(\pi^{-1}(p)\right)=K$ for all $p \in \alpha X \backslash X$. Thus $E=\varnothing$ (as long as $K$ is not a singleton) and so $\Gamma_{f}=\varnothing$. We have

$$
\begin{aligned}
e X \backslash e(X) & =\Delta_{f}=\left\{(p, q) \mid p \in(\alpha X \backslash X) \backslash E \text { and } q \in \bar{f}\left(\pi^{-1}(p)\right)\right\} \\
& =\{(p, q) \mid p \in \alpha X \backslash X \text { and } q \in K\}=(\alpha X \backslash X) \times K .
\end{aligned}
$$

5. The $n$-complementation property. In this section we identify a class of spaces each having precisely the same set of remainders. This will extend the same type of theorem which appears in Blakley, Gerlits and Magill [1]. For this section we 
assume all spaces to be separable metric (as well as locally compact). In this context then, a continuum is a compact connected space and a generalized continuum is a connected space. A conditionally compact subset of a space is a subset whose closure is compact. A space $X$ has the $n$-complementation property [4] if for each member $F \in \mathcal{K}_{X}$ there is a member $K \in \mathcal{K}_{X}$ with $F \subset K$ and $X \backslash K$ has exactly $n$ nonconditionally compact components.

LEMMA 5.1. Suppose $Z$ is a locally connected continuum, $U$ is an open connected subset of $Z$, and $C \subset U$ is compact and connected. Then there is a locally connected continuum $K$ such that $C \subset K \subset U$.

Proof. The set $U$ is locally arcwise connected (Whyburn [13, 5.3, p. 38]). There is a mapping $f$ from the Cantor discontinuum onto $C$; and by Theorem 5, p. 253 of Kuratowski [8], $f$ has a continuous extension mapping the closed interval into $U$. The required set $K$ is the image of the interval under this extension.

LEMMA 5.2. Suppose $Y$ is a locally connected continuum, and $p \in Y$. There exists a sequence $P_{0}, P_{1}, \ldots$ of locally connected continua such that

(i) $p \in Y \backslash P_{i}, i=1,2,3, \ldots$;

(ii) $Y \backslash\{p\}=\cup P_{i}$;

(iii) given a neighborhood $U$ of $p$, there is an $N$ so that $P_{i} \subset U$ for all $i>N$.

Proof. Let $\left\{G_{j}\right\}_{j=0}^{\infty}$ be an open connected base at $p$ so that $G_{0}=Y$, and $G_{m} \supset \bar{G}_{m+1}$ for all $m=0,1, \ldots$ The set $G_{m} \backslash \bar{G}_{m+1}$ is open and so has a finite number of components $C_{1}^{m}, C_{2}^{m}, \ldots, C_{l(m)}^{m}$. Then each $\bar{C}_{j}^{m}$ is a compact connected set, and is contained in some component of $G_{m-1} \backslash \bar{G}_{m+2}$. By 5.1 there is a locally connected continuum $K_{j}^{m}$ so that $\bar{C}_{j}^{m} \subset K_{j}^{m} \subset G_{m-1} \backslash \bar{G}_{m+2}$.

Now define the sequence $P_{1}, P_{2}, \ldots$ by taking $K_{1}^{1}, K_{2}^{1}, \ldots, K_{l(1)}^{1}$ to be the first $l(1)$ terms, and then $K_{1}^{2}, K_{2}^{2}, \ldots, K_{l(2)}^{2}$ to be the next $l(2)$ terms and so on.

To verify that $\left\{P_{i}\right\}$ satisfies the conclusions of the lemma, note first that if $p$ were a member of say $K_{j}^{m}$, then $p \in G_{m-1} \backslash \bar{G}_{m+2}$, a contradiction since $p \in G_{m+2}$. Thus $p \in Y \backslash P_{j}$. Next suppose $x \in Y$, and $x \neq p$. Let $m$ be the smallest integer for which $x \notin \bar{G}_{m+2}$; then $x \in G_{m-1} \backslash \bar{G}_{m+2}$ and hence is in some $\bar{C}_{j}^{m} \subset K_{j}^{m}$.

Finally, if $U$ is a neighborhood of $p$, choose $m$ so that $\bar{G}_{m-1} \subset U$. It is clear that all $P_{i}$ beyond $K_{j}^{m+2}$ are contained in $\bar{G}_{m-1}$.

Let $L_{k}=\{(x, y) \in \mathbf{R} \times \mathbf{R} \mid y=x / k, x \geqslant 0\}$ and define

$$
T_{n}=\bigcup_{k=1}^{n} L_{k} .
$$

THEOREM 5.3. Suppose $X$ is a locally connected generalized continuum with the $n$-complementation property. Then there is a compact continuous map $F: T_{n} \rightarrow F\left(T_{n}\right)$ $=X$ of $T_{n}$ onto $X$.

Proof. The space $X$ has an $n$-point compactification $\alpha X$ which is locally connected and metric [4, 3.4 and 2.2]. Let $\alpha X \backslash X=\left\{z_{1}, z_{2}, \ldots, z_{n}\right\}$. Each $z_{k}$ is a noncutpoint of $\alpha X$, so for each $k$ there is an open set $V_{k}$ so that

$$
R_{k}=\left\{z_{1}, z_{2}, \ldots, z_{k-1}, z_{k+1}, \ldots, z_{n}\right\} \subset V_{k},
$$


$z_{k} \notin \bar{V}_{k}$, and $\alpha X \backslash V_{k}$ is connected. Now apply Lemma 5.1 with $Z=\alpha X, U=$ $\alpha X \backslash R_{k}$, and $C=\alpha X \backslash V_{k}$ to conclude the existence of a locally connected continuum $Y_{k}$, with $\alpha X \backslash V_{k} \subset Y_{k} \subset \alpha X \backslash R_{k}$.

For $z_{k}=p$, let $\left\{P_{i}^{k}\right\}$ be the sequence of locally connected continua whose existence is insured by Lemma 5.2.

Define subsets $L_{k, m} \subset T_{n}$ as follows:

$$
L_{k, m}=\left\{(x, y) \in L_{k} \mid m \leqslant x \leqslant m+1\right\}
$$

for $k=1,2, \ldots, n$ and $m=0,1,2, \ldots$.

Each $L_{k, m}$ is a homeomorph of the unit interval, so there are continuous functions $f_{k, 2 m+1}$ mapping $L_{k, 2 m+1}$ onto $P_{m}^{k}$ for each $k=1,2, \ldots, n$, and $m=$ $0,1,2, \ldots$.

Suppose $U_{1}, U_{2}, \ldots, U_{n}$ are disjoint open connected neighborhoods of $z_{1}, \ldots, z_{k}$, respectively. Then each $W_{k}=U_{k} \backslash\left\{z_{k}\right\}$ is connected. Otherwise $W_{k}$ would have at least two nonconditionally compact (in $X$ ) components, say $A_{1}$ and $A_{2}$. There is a compact set $K \subset X$ such that $X \backslash K \subset \cup_{1}^{n} W_{i}$, and $X \backslash K$ has exactly $n$ nonconditionally compact components. But this is a contradiction, since both $A_{1}$ and $A_{2}$ would contain components, necessarily nonconditionally compact, of $X \backslash K$.

Thus for each $z_{k} \in \alpha X \backslash X$, there is an open connected base $\left\{U_{i}^{k}\right\}$ such that each $W_{i}^{k}=U_{i}^{k} \backslash\left\{z_{k}\right\}$ is open (in $X$ ), locally connected, and connected, and hence arcwise connected.

It follows from Lemma 5.2 that for each $k$, there is a sequence $\{n(k ; j)\}_{j=1}^{\infty}$ such that $n(k ; j+1) \geqslant n(k ; j)$, and $P_{i}^{k} \subset W_{j}^{k}$ for $i \geqslant n(k ; j)$.

For each $k=1,2, \ldots, n$, define a collection of maps $f_{k, 2 m+2}$ as follows:

(i) for $0 \leqslant m<n(k ; 1)$, define $f_{k, 2 m+2}$ to be a homeomorphism of $L_{2 m+2}$ into $X$; ( $X$ is arcwise connected.)

(ii) for $n(k ; j) \leqslant m<n(k ; j+1)$, we have $P_{m}^{k}$ and $P_{m+1}^{k} \subset W_{j}^{k}$, which is arcwise connected. Thus for each such $m$, there is a homeomorphism $f_{k, 2 m+2}$ of $L_{2 m+2}$ into $W_{j}^{k}$ such that

$$
f_{k, 2 m+2}(2 m+2,(2 m+2) / k)=f_{k, 2 m+1}(2 m+2,(2 m+2) / k)
$$

and

$$
f_{k, 2 m+2}(2 m+3,(2 m+3) / k)=f_{k, 2 m+3}(2 m+3,(2 m+3) / k) ;
$$

(iii) finally, let $p$ be an arbitrary point in $X$, and define $f_{k, 0}$ to be a homeomorphism of $L_{k, 0}$ into $X$ with $f_{k, 0}(0,0)=p$ and

$$
f_{k, 0}(1,1 / k)=f_{k, 1}(1,1 / k) \text {. }
$$

Now define $F: T_{n} \rightarrow F\left(T_{n}\right)=X$ by

$$
F(z)= \begin{cases}f_{k, 2 m}(z), & z \in L_{k, 2 m}, \\ f_{k, 2 m+1}(z), & z \in L_{k, 2 m+1} .\end{cases}
$$

Note that $F$ is well defined and maps $T_{n}$ continuously onto $X$ since the mappings $f_{k, m}$ agree on the common parts of their domains. 
To show that $F$ has no singular points, let $\left\{t_{i}\right\}$ be a sequence in $T_{n}$ which has no convergent subsequence. This means that there are at most a finite number of terms of $\left\{t_{i}\right\}$ in any $L_{k, m}$. It follows that $\left\{F\left(t_{i}\right)\right\}$ cannot have a convergent subsequence in $X$. Or in other words, $F$ is compact.

It follows from [6, Theorem 2] that if $X$ has the $n$-complementation property, there is a compact map of $X$ onto $T_{n}$. Thus from Corollary 2.2, we have

THEOREM 5.4. The remainders of all locally connected generalized continua with the n-complementation property are identical.

Corollary 5.5 (Blakley, Gerlits and Magill). The remainders of all ringed spaces are identical.

Proof. A ringed space is precisely a locally connected generalized continuum with the 1-complementation property.

6. Compact mappings and manifolds. The results in this section are seemingly unconnected with singular maps. However, the ideas here were originally obtained using results on singular mappings. We then were able to devise the more elementary proofs which we have here. Because of this original relationship with singular mappings and because the results seem to be of some interest in their own right, we include them here. We are indebted to our colleagues Harvey Charlton and Steve Schecter for their assistance in obtaining these alternate proofs.

We will say that a map $f: X \rightarrow Y$ is $1-1$ at $x \in X$ if $f^{-1}(f(x))=\{x\}$. A space $X$ is strongly locally connected at $x$ if $x$ is not isolated and for each neighborhood $U$ of $x$ there is an open neighborhood $V$ of $x$ with $V \subseteq U$ and with $V \backslash\{x\}$ connected. $S^{1}$ will denote the circle with its usual topology.

THEOREM 6.1. If $X$ is compact and strongly locally connected at $x$ then no map $f$ : $X \Rightarrow S^{1}$ can be 1-1 at $x$.

Proof. If $f$ were $1-1$ at $x$, then letting $Z=X \backslash\{x\}$ and $\mathbf{R}^{1}=S^{1} \backslash\{f(x)\}$ we would, by restriction, have a map $g: Z \Rightarrow \mathbf{R}^{1}$. Now $g$ is compact since $f$ is. Thus for any $p \in \mathbf{R}^{1}$ we have $g^{-1}(p)$ is a compact subset of $Z$, and, hence, of $X$. If we let $U=X \backslash g^{-1}(p)$, there is an open neighborhood $V$ of $x$ with $V \subseteq U$ and $V \backslash\{x\}$ connected. $g(V \backslash\{x\})$ is connected and hence must be contained in $[p, \infty)$ or in $(-\infty, p]$. We have

$$
\mathbf{R}^{1}=g(Z)=g(Z \backslash V) \cup g(V \backslash\{x\})
$$

and, since $Z \backslash V=X \backslash V, g(Z \backslash V)$ is compact. This is not possible, since we would have $\mathbf{R}^{1}$ contained in the union of this compact set and one of the rays $[p, \infty)$ or $(-\infty, p]$.

By a compact $n$-manifold we mean a compact Hausdorff space $M^{n}$, each point having a neighborhood homeomorphic to $\mathbf{R}^{n}$. Since such a space is strongly locally connected at each of its points if $n>1$ we have

COROllary 6.2. If $f: M^{n} \Rightarrow S^{1}$ and $n>1$ then $f$ is not 1-1 at any point of $M^{n}$. 
COROLlaRY 6.3. If $X$ is compact, connected, and strongly locally connected at each of its points and $f: X \rightarrow \mathbf{R}^{1}$ then $f$ is not 1-1 at any point of $X$ except possibly at its absolute maxima and minima.

Proof. Suppose $f: X \Rightarrow[m, M]$. We can obtain a map of $X$ onto $S^{1}$ by identifying $m$ and $M$.

We cannot substitute any other sphere for $S^{1}$ and get an analogous result. We need the following lemma to establish this.

LEMMA 6.4. There is a compact map $f: \mathbf{R}^{n} \Rightarrow \mathbf{R}^{m}$ if $n>m>1$.

Proof. We show that the lemma is true for $n=m+1$. The full generality is then obtained by appropriately composing such maps. Let $\mathbf{R}_{+}^{m}=\left\{\left(x_{1}, \ldots, x_{m}\right) \in\right.$ $\left.\mathbf{R}^{m} \mid x_{m} \geqslant 0\right\}$ and define $g: \mathbf{R}^{m+1} \Rightarrow \mathbf{R}_{+}^{m}$ by

$$
g\left(\left(x_{1}, \ldots, x_{m}, x_{m+1}\right)\right)=\left(x_{1}, \ldots, x_{m-1}, \sqrt{x_{m}^{2}+x_{m+1}^{2}}\right),
$$

and define $h: \mathbf{R}_{+}^{m} \Rightarrow \mathbf{R}^{m}$ by

$$
\begin{aligned}
& h\left(\left(p_{1}, \ldots, p_{m}\right)\right) \\
& \quad=\left(p_{1}, \ldots, p_{m-2}, 2 p_{m-1} p_{m} / \sqrt{p_{m-1}^{2}+p_{m}^{2}},\left(p_{m}^{2}-p_{m-1}^{2}\right) / \sqrt{p_{m-1}^{2}+p_{m}^{2}}\right) .
\end{aligned}
$$

Letting $f=h \circ g$ completes the construction. In effect (when $m=2$ ), the map $g$ "circularly projects" $\mathbf{R}^{3}$ onto the half-plane in $\mathbf{R}^{2}$ of those points whose second coordinate is nonnegative. $h$ then "opens up" this half-plane onto all of $\mathbf{R}^{2}$. The composition is clearly compact.

THEOREM 6.5. If $n>m>1$ there is a map $f: M^{n} \Rightarrow S^{m}$ which is 1-1 at some point ( $M^{n}$ is any compact $n$-manifold).

Proof. Let $p \in M^{n}$ and define a map $g: M^{n} \backslash\{p\} \Rightarrow \mathbf{R}^{n}$ as follows: Let $B$ be a compact neighborhood of $p$ in $M^{n}$ with a homeomorphism $h: B \Rightarrow \overline{\mathbf{B}^{n}}=\{\bar{x} \in$ $\left.\mathbf{R}^{n} \mid\|\bar{x}\| \leqslant 1\right\}$, for which $h(p)=\overline{0}$. Let $k: \overline{\mathbf{B}^{n}} \backslash\{\overline{0}\} \Rightarrow \mathbf{R}^{n} \backslash\{\overline{0}\}$ be defined by $k(\bar{x})=$ $\bar{x}(1-\|\bar{x}\|) /\|\bar{x}\|^{2}$. Finally, define $g$ by

$$
g(y)= \begin{cases}\overline{0} & \text { if } y \notin B, \\ k \circ h(y) & \text { if } y \in B .\end{cases}
$$

By composing $g$ with the map given in the preceding lemma we get a compact mapping $\bar{f}: M^{n} \backslash\{p\} \Rightarrow \mathbf{R}^{m}$. By a theorem of Whyburn [15] there is an extension $f$ : $M^{n} \Rightarrow S^{m}=\mathbf{R}^{m} \cup\{\infty\}$ which carries $p$ to $\infty . f$ is $1-1$ at $p$.

\section{REFERENCES}

1. G. R. Blakley, J. Gerlits and K. D. Magill, Jr., A class of spaces with identical remainders, Studia Sci. Math. Hungar. 6 (1971), 117-122.

2. George L. Cain, Jr., Compact and related mappings, Duke Math. J. 33 (1966), 639-645.

3. __ Mappings with prescribed singular sets, Nieuw. Arch. Wisk. (3) 17 (1969), 200-203.

4. (1980), 311-315.

5. __ Continuous preimage of spaces with finite compactifications, Canad. Math. Bull. (to appear). 6. Richard E. Chandler and Fu-Chien Tzung, Remainders in Hausdorff compactifications, Proc. Amer. Math. Soc. 70 (1978), 196-202. 
7. Eng Ung Choo, Accumulation points on continuous real-valued functions and compactifications, Canad. Math. Bull. 20 (1977), 47-52.

8. K. Kuratowski, Topology, Vol. II, Academic Press, New York and London, 1968.

9. Peter A. Loeb, A minimal compactification for extending continuous functions, Proc. Amer. Math. Soc. 18 (1967), 282-283.

10. K. D. Magill, Jr., More on remainders of spaces in compactifications, Bull. Acad. Polon. Sci. Sér. Sci. Math. Astronom. Phys. 18 (1970), 449-451.

11. A. K. Steiner and E. F. Steiner, Compactifications as closures of graphs, Fund. Math. 63 (1968), 221-223.

12. Graph closures and metric compactifications of N, Proc. Amer. Math. Soc. 25 (1970), . 593-597.

13. G. T. Whyburn, Analytic topology, Amer. Math. Soc. Colloq. Publ., vol. 28, Amer. Math. Soc., Providence, R. I., 1963.

14. , Compactification of mappings, Math. Ann. 166 (1966), 168-174.

15. __ Dynamic topology, Amer. Math. Monthly 77 (1970), 556-570.

Department of Mathematics, Georgia Institute of Technology, Atlanta, Georgia 30332

Department of Mathematics, North Carolina State University, Raleigh, North Carolina 27650 